\title{
Electronic Structure of Cobalt Nanocrystals Suspended in Liquid
}

\author{
Hongjian Liu, ${ }^{\dagger}$ Jinghua Guo, ${ }^{*, \ddagger}$ Yadong Yin,, Andreas Augustsson,,,$\| l$ \\ Chungli Dong,,$\neq, \perp$ Joseph Nordgren," Chinglin Chang, ${ }^{\perp}$ Paul Alivisatos, $, \$, \#$ \\ Geoff Thornton, ${ }^{\nabla}$ D. Frank Ogletree,,${ }^{\dagger} \S$ Felix G. Requejo, ${ }^{\circ}$ Frank de Groot, ${ }^{\star}$ and \\ Miquel Salmeron ${ }^{\star}, t, \S$
}

\begin{abstract}
Materials Sciences Division, Lawrence Berkeley National Laboratory, Berkeley, California 94720, Advanced Light Source, Lawrence Berkeley National Laboratory, Berkeley, California 94720, The Molecular Foundry, Lawrence Berkeley National Laboratory, Berkeley, California 94720, Department of Physics, Uppsala University, Box 530, 75121 Uppsala, Sweden, Department of Physics, Tamkang University, Tamsui, Taiwan 251, R.O.C., Department of Chemistry, University of California, Berkeley, California 94720, London Centre for Nanotechnology, Chemistry Department, University College London WCIH OAJ, United Kingdom, Departamento de Física, FCE, UNLP-INIFTA (CONICET), Argentina, Department of Chemistry, Utrecht University, The Netherlands
\end{abstract}

Received March 13, 2007; Revised Manuscript Received May 24, 2007

\begin{abstract}
The electronic structure of cobalt nanocrystals suspended in liquid as a function of size has been investigated using in situ X-ray absorption and emission spectroscopy. A sharp absorption peak associated with the ligand molecules is found that increases in intensity upon reducing the nanocrystal size. X-ray Raman features due to $d-d$ and to charge-transfer excitations of ligand molecules are identified. The study reveals the local symmetry of the surface of $\epsilon$-Co phase nanocrystals, which originates from a dynamic interaction between Co nanocrystals and surfactant + solvent molecules.
\end{abstract}

Advances in the synthesis of particles of nanometer dimensions, narrow size distribution, and controlled shape have generated interest because of the potential to create novel materials with tailored physical and chemical properties. ${ }^{1-3}$ New properties arise from quantum confinement effects and from the increasing fraction of surface atoms with unique bonding and geometrical configurations. Co nanocrystals display a wealth of size-dependent structural, magnetic, electronic, and catalytic properties. The challenges in making isolated Co nanocrystals are to overcome the large attractive forces between the nanoparticles, due to surface tension and van der Waals interactions that tend to aggregate them.

* Corresponding authors. E-mail: mbsalmeron@lbl.gov (M.S.) and jguo@lbl.gov (J.H.G.).

Materials Sciences Division, Lawrence Berkeley National Laboratory.

\#dvanced Light Source, Lawrence Berkeley National Laboratory.

$\S$ The Molecular Foundry, Lawrence Berkeley National Laboratory. Y. Yin is now at the Department of Chemistry, University of California, Riverside.

"Department of Physics, Uppsala University.

${ }^{\perp}$ Department of Physics, Tamkang University.

\# Department of Chemistry, University of California, Berkeley.

$\nabla$ London Centre for Nanotechnology, Chemistry Department, University College London.

Departamento de Física, FCE, UNLP-INIFTA (CONICET).

- Department of Chemistry, Utrecht University.
Using appropriate surfactants, however, Co nanocrystals could be grown with controlled shapes and sizes: either spheres or disks in a surfactant mixture. ${ }^{3-5}$ It was found that disks of hcp-Co are obtained in a binary surfactant mixture at early times after injection of the precursor and spontaneously transform to the more thermodynamically stable spheres of $\epsilon$-Co after heating for a sufficient period of time. ${ }^{6}$

A fundamental understanding of the growth and properties of the nanocrystals would greatly benefit from a detailed information of their electronic structure as a function of size and of the presence and nature of the molecules bound to their surface. Because the Co nanocrystals are extremely reactive and oxidize easily, it is important to use techniques that can interrogate the particles in their growth environment so that their electronic and chemical structure can be followed during growth and during catalytic reactions.

We report an electronic structure study of Co nanocrystals with a narrow size distribution suspended in 1,2-dichlorobenzene $\left(\mathrm{C}_{6} \mathrm{H}_{4} \mathrm{Cl}_{2}\right)$ using in situ photon-in/photon-out spectroscopies, including X-ray absorption (XAS), ${ }^{7} \mathrm{X}$-ray emission (XES), and resonant inelastic X-ray scattering (RIXS). ${ }^{8,9}$ These techniques are element selective as they 
involve core atomic levels and thus can probe the local electronic structure of selected species in a complex system. ${ }^{10-12} \mathrm{We}$ found particle size effects arising from interactions with surfactant and solvent molecules at the particle surface.

Cobalt nanocrystals were synthesized by decomposing the carbonyl precursor, $\mathrm{Co}_{2}(\mathrm{CO})_{8}$, in 1,2-dichlorobenzene at a temperature around its boiling point $\left(\sim 182{ }^{\circ} \mathrm{C}\right) .{ }^{3-5}$ Oleic acid, $\mathrm{CH}_{3}\left(\mathrm{CH}_{2}\right)_{7} \mathrm{CH}=\mathrm{CH}\left(\mathrm{CH}_{2}\right)_{7} \mathrm{CO}_{2} \mathrm{H}$, and trioctylphosphine oxide, $\left(\mathrm{CH}_{3}\left(\mathrm{CH}_{2}\right)_{7}\right)_{3} \mathrm{P}=\mathrm{O}$ (TOPO), were used to control the growth and to provide a protective surfactant capping. In a volume of $18 \mathrm{~mL}$ solution, the amount of TOPO was $0.1 \mathrm{~g}$. The size of Co nanocrystals depends mainly on the amount of oleic acid, while TOPO has a much weaker interaction with the cobalt surface. Monodispersed Co nanocrystals with sizes of $3,4,5,6$, and $9 \mathrm{~nm}( \pm 0.65 \mathrm{~nm})$ were prepared by simply adjusting the amount of oleic acid used in the synthesis. The size of the nanocrystals was determined by transmission electron microscopy (TEM). Samples of liquid suspensions were encapsulated in a small cell inside a glove box under argon gas before transfer to beamline 7.0 of the Advanced Light Source for X-ray spectroscopic experiments. ${ }^{13}$ The cell was sealed with a silicon nitride window $100 \mathrm{~nm}$ thick. ${ }^{14}$ Incident and emitted X-ray photons penetrated through this window. The X-ray absorption spectra were recorded in total fluorescence yield mode using a channeltron. X-ray emission spectra were obtained using a high-resolution grazing-incidence grating spectrometer. The energy resolution was $0.2 \mathrm{eV}$ for XAS and $0.7 \mathrm{eV}$ for XES measurements.

The Co L-edge XAS of nanocrystals are shown in Figure 1, along with $\mathrm{Co}$ metal, $\mathrm{CoCl}_{2}, \mathrm{Co}_{3} \mathrm{O}_{4}$, and $\mathrm{CoO}$ reference spectra. The spectra contain two regions separated by $16.0 \mathrm{eV}$ due to the core-level spin orbital splitting of the $2 \mathrm{p}_{3 / 2}$ and $2 \mathrm{p}_{1 / 2}$ orbitals. The high branching ratio of $\mathrm{L}_{2} /\left(\mathrm{L}_{2}+\mathrm{L}_{3}\right)$ in the $\mathrm{CoO}$ spectrum indicates a saturation effect due to the fluorescence-detection method, and for that reason, the profile of the $\mathrm{L}_{3}$-edge is used for comparison. The saturation effect is not a significant problem for Co nanocrystals as they are dispersed in the suspension. The most notable feature in the spectra of the Co nanocrystals is the new absorption peak, $\mathrm{A} 2$, at $\sim 6 \mathrm{eV}$ above the main absorption edge that is absent in the Co metal, $\mathrm{CoO}, \mathrm{Co}_{3} \mathrm{O}_{4}$, and $\mathrm{CoCl}_{2}$. Because the precursors in the synthesis materials contain only the elements $\mathrm{Co}, \mathrm{C}, \mathrm{O}$, and $\mathrm{Cl}$, no other absorption lines are expected near the Co L-edges.

We investigated the origin of the A2 peak using the X-ray emission spectrometer to measure XAS spectra in partial fluorescence-detection mode. No emission changes associated with this peak were observed within a window of 50$100 \mathrm{eV}$ centered at the Co L-edge $(780 \mathrm{eV}), \mathrm{O}$ K-edge (525 eV), C K-edge (275 eV), or Cl L-edge (170 eV). Thus the fluorescence yield of this peak must be due to photons with energy below $120 \mathrm{eV}$, which are detected by the channeltron but not the spectrometer. As we discuss next, we believe that this peak is due to a metal-to-ligand charge transfer (MLCT) to unoccupied ligand orbitals involved in $\pi$ back-bonding to the Co metal.

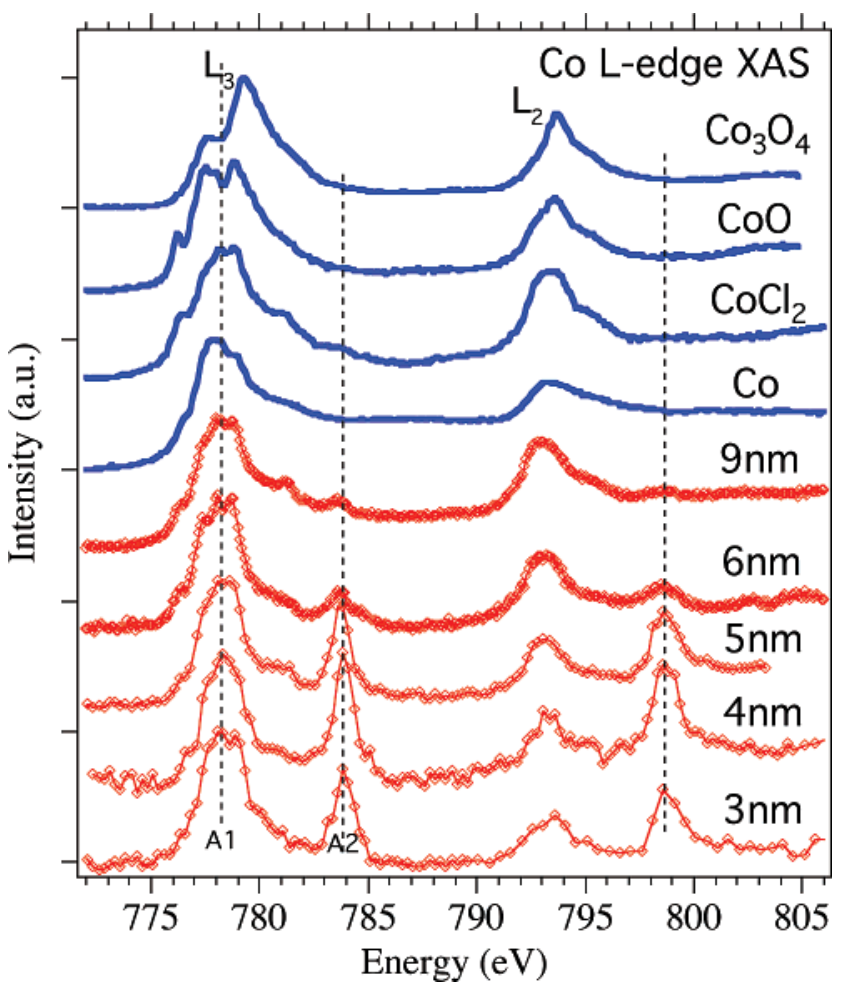

Figure 1. (a) Co $2 p$ X-ray absorption spectra of cobalt nanoparticles with diameters from 3 to $9 \mathrm{~nm}$ in a 1,2-dichlorobenzene liquid suspension. The top four spectra correspond to $\mathrm{Co}_{3} \mathrm{O}_{4}, \mathrm{CoO}, \mathrm{CoCl}_{2}$, and cobalt metal. The peak at $784 \mathrm{eV}\left(\mathrm{A}_{2}\right.$, dashed line $)$ in the X-ray absorption spectra of the nanocrystals is absent in $\mathrm{CoO}$ and $\mathrm{Co}$ metal. This peak is attributed to a metal-to-ligand charge transfer (MLCT).

The first discussion on $\pi$ back-bonding in metal L-edge XAS was given in a study of bimetallic cyanides of the Prussian blue family. ${ }^{15}$ A similar absorption satellite was found for $\mathrm{K}_{2} \mathrm{Ni}(\mathrm{CN})_{4}$ in comparison with $\mathrm{Ni}$ metal or $\mathrm{NiO}$ systems, where MLCT transitions to unoccupied ligand orbitals were identified. ${ }^{16,17}$ A similar MLCT feature was observed in $\mathrm{K}_{3} \mathrm{Co}(\mathrm{CN})_{6}$ that adds support to our assignment. ${ }^{18}$ The A2 satellite peak in Figure 1 is then assigned to MLCT transitions between Co and the oleic acid or 1,2-dichlorobezene. The MLCT satellite peak starts to appear in the nanocrystals of $9 \mathrm{~nm}$ and shows an increasing intensity when the diameter decreases, as expected from the increasing proportion of Co-surfactant molecular interactions.

We can exclude residual unreacted Co carbonyls as contributing to this peak because they should be present in similar amounts in the Co nanocrystal suspensions with diameters of 3-9 $\mathrm{nm}$. Also, the metal carbonyls do not have a strong peak at energies above the $\mathrm{L}_{3}$ (and $\mathrm{L}_{2}$ edge), as shown for example in the spectra of $\mathrm{Mn}_{2}(\mathrm{CO})_{10}$ measured by Ruhl and Hitchcock. ${ }^{19}$ This suggests that the $\mathrm{CO} \pi$ orbitals have a different energy ordering with respect to the $3 \mathrm{~d}$ band that seems not to yield an additional peak; probably the $\mathrm{CO} \pi^{*}$ peaks are mixed within the L edge multiplet. This is also seen in heme-like complexes, as discussed by Hocking et al. ${ }^{20}$

Figure 2a shows the RIXS spectra from $6 \mathrm{~nm}$ nanocrystals recorded at the selected energies indicated by arrows in the 

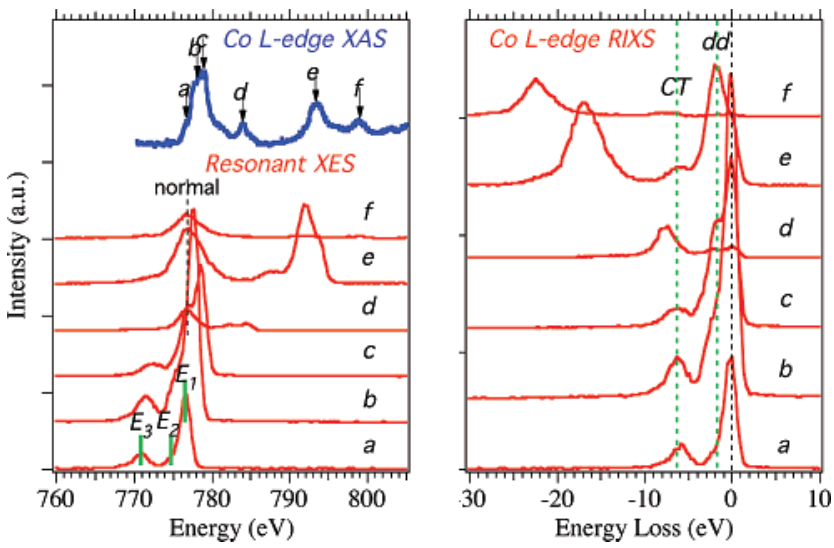

Figure 2. (a) Resonant X-ray emission spectra recorded at selected excitation energies (marked by arrows in the XAS at the top) of the Co $2 p$ threshold for cobalt nanocrystals with a diameter of $6 \mathrm{~nm}$. (b) Resonant inelastic X-ray scattering spectra on an energyloss (Raman) scale.

XAS. The intensity has been normalized to the incoming photon flux. The spectra comprise three contributions: normal X-ray emission, elastic, and inelastic X-ray scattering. Normal X-ray emission is dominant at excitation well above the Co L-edge absorption threshold. The normal emission appears at constant photon energy, $778 \mathrm{eV}$ for the $\mathrm{L}_{3}$ edge (indicated by dash line), and independent of changes in the excitation energy. The X-ray scattering contribution includes an elastic peak at the same energy as the incident photons and inelastic peaks at lower energies that are regarded as $\mathrm{X}$-ray Raman scattering. Strong resonance effects are observed in the scattering intensity as incident energy changes from a to $f$. The same data are plotted on an energy-loss scale (Figure 2b). The elastic peak (at the origin) corresponds to the final state of $\mathrm{X}$-ray scattering process equal to the ground state, while the loss peaks correspond to final excited states near the ground state. The peak at $\sim-2 \mathrm{eV}$ is attributed to transitions where the final configuration contains quartetquartet and quartet - doublet $\mathrm{d}-\mathrm{d}$ excitations of the crystal field split d-levels. ${ }^{21,22}$ The relative intensity of the peak shows a notable resonance effect with incident energy. Another set of Raman peaks occurs at $-6.7 \mathrm{eV}$, which corresponds to the ligand $\mathrm{O} 2 \mathrm{p}-$ Co $3 \mathrm{~d}$ charge-transfer excitations.

To understand these spectroscopic observations, the singleimpurity Anderson model with full multiplet effects has been applied. The calculations were performed in the so-called charge-transfer ligand field multiplet theory developed by Thole, following Cowan and Butler. ${ }^{23-25}$ This approach takes into account all the electronic interactions and the spinorbit coupling on any shell and treats the geometrical environment of the absorbing atom through crystal field potential. ${ }^{7}$ In the simplest formulation, a pure $3 \mathrm{~d}^{n}$ configuration is attributed to the ground state of the $3 \mathrm{~d}$ transition ion. One then calculates the transitions between the $2 \mathrm{p}^{6} 3 \mathrm{~d}^{n}$ ground state toward the $2 \mathrm{p}^{5} 3 \mathrm{~d}^{n+1}$ excited states. Chargetransfer effects are included by adding additional configurations, for example adding $3 \mathrm{~d}^{7}+3 \mathrm{~d}^{8} \mathrm{~L}$ to describe ligandto-metal charge transfer, $3 \mathrm{~d}^{7}+3 \mathrm{~d}^{6} \mathrm{~L}$ to describe metal-toligand charge transfer, or a combination of both channels.

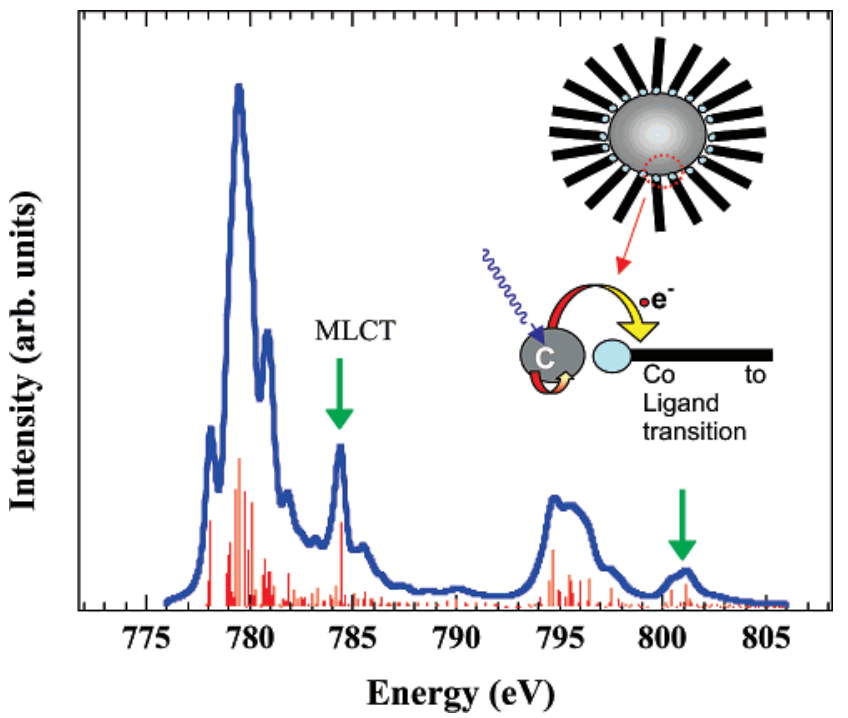

Figure 3. Calculated Co L-edge absorption spectrum from the single-impurity Anderson model. The multiplets have been broadened with the experimental resolution function. The insert shows the chemicals involved in the synthesis of Co nanocrystals from dicobalt carbonyl $\left(\mathrm{Co}_{2}(\mathrm{CO})_{8}\right)$. It also illustrates a charge transfer from Co nanocrystals to ligand molecules.

In Co metal, the ground state is $4 \mathrm{~s}^{2} 3 \mathrm{~d}^{7},{ }^{26}$ while for $\mathrm{CoO}$, one uses the ground state configuration $\left[3 \mathrm{~d}^{7}+3 \mathrm{~d}^{8} \mathrm{~L}^{-1}\right]\left(\mathrm{L}^{-1}\right.$ denotes a hole in the ligand level). Although this explained the $\mathrm{CoO}$ spectrum quite well,,$^{22}$ the $\left[3 \mathrm{~d}^{7}+3 \mathrm{~d}^{8} \mathrm{~L}^{-1}\right]$ never yielded a significant satellite contribution, as seen in Co nanocrystals (in Figure 1). The only known octahedral systems with large satellites are cyanide complexes, where large satellites are caused by $\pi$ back-bonding, i.e., $\left[3 \mathrm{~d}^{7}+\right.$ $\left.3 \mathrm{~d}^{6} \mathrm{~L}\right]$.

Figure 3 shows the simulations including metal-to-ligand charge transfer (MLCT) effects. The calculations describe the ground state as $\left[3 \mathrm{~d}^{7}+3 \mathrm{~d}^{6} \mathrm{~L}\right]$ with the MLCT energy $\Delta_{\pi}$ of $-3.0 \mathrm{eV}$. This yields a ground state that has $38 \% 3 \mathrm{~d}^{7}$ and $62 \% 3 \mathrm{~d}^{6} \mathrm{~L}$, where it is noticed that the main $\mathrm{L}_{3}$ edge still looks similar to that of the $3 \mathrm{~d}^{7}$ ground state found in $\mathrm{CoO}$. The main structure is then a $\left[2 p^{5} 3 d^{8}+2 p^{5} 3 d^{7} L\right]$ bonding combination and the satellite is the antibonding part. It is worth noticing that the MLCT acts mainly on the $t_{2 g}$ electrons. The optimal simulation of the spectral shape was obtained if mainly the Co $3 \mathrm{~d}_{x y}$-orbital was mixed with ligand orbitals in $D_{4 H}$ symmetry. We use $\mathrm{t}_{x y}=3.0 \mathrm{eV}$ and $\mathrm{t}_{x z}, \mathrm{t}_{y z}=$ $1.0 \mathrm{eV}$. Assuming a Co surface ion bonded to a ligand in the $z$-direction, the $x y$-orbital (with $B_{2}$ symmetry) is the orbital that can donate its electrons to empty $\pi$-states. This implies that the surface Co atoms in nanocrystals are aligned in an ordered fashion to connect the ligands perpendicularly to the nanocrystal surface, which gives an explanation of why no stacking faults ${ }^{4}$ caused by the sliding atomic planes were observed in $\epsilon$-Co nanocrystals in contrast to hcp-Co. Also, the surface Co atoms interact less with the ligands in the larger Co nanocrystals, as indicated by the reduced intensity of X-ray absorption satellites.

Figure 4a shows RIXS spectra as a function of Co nanocrystal size at the excitation corresponding to $2 \mathrm{p}_{3 / 2}$ absorption edge $(778 \mathrm{eV})$. The $-2 \mathrm{eV}$ peak $(\mathrm{d}-\mathrm{d}$ excitation) 

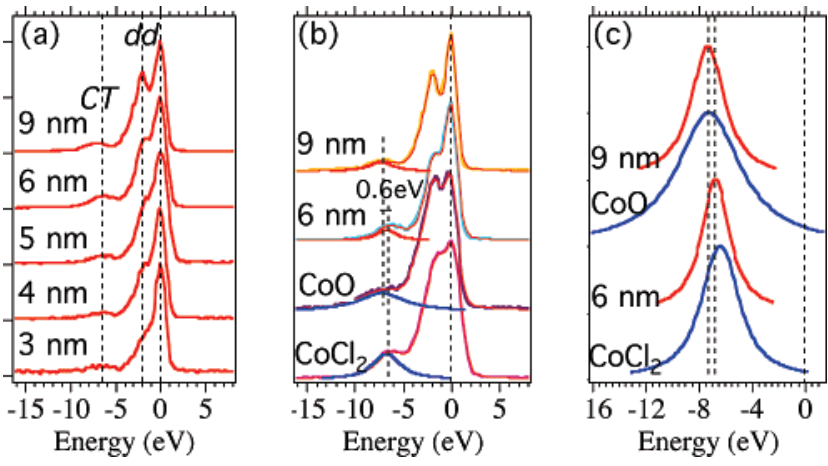

Figure 4. (a) Energy-loss features of $\mathrm{Co}_{3}$-edge RIXS for $\mathrm{Co}$ nanocrystals of different diameters. (b) RIXS spectra of $\mathrm{CoO}$ and $\mathrm{CoCl}_{2}$ compared with that for nanoparticles of 6 and $9 \mathrm{~nm}$ diameter. The nanoparticles spectra are fitted by a sum of Voigt function peak shapes. The excellent quality of the fit makes it undistinguishable from the experimental spectrum. (c) The charge-transfer peaks, at $-7.3 \mathrm{eV}$ for the $9 \mathrm{~nm}$ and $-6.7 \mathrm{eV}$ for the $6 \mathrm{~nm}$ nanocrystals, respectively, coincide in position with those observed from $\mathrm{CoO}$ and $\mathrm{CoCl}_{2}$.

increases its intensity with nanocrystals size. The unchanged peak position suggests that the local symmetry and surrounding environment in Co nanocrystals remains unchanged and is similar to that of the $\epsilon$-Co phase, as previously determined. The increase of intensity with nanocrystals size is in line with the decreasing fraction of surface Co atoms.

The intensity of the charge-transfer peak at $\sim-6$ to $-7 \mathrm{eV}$ increases with nanocrystal size from 3 to $6 \mathrm{~nm}$ and decreases thereafter. Interestingly, there is also a peak shift from the $9 \mathrm{~nm}$ to the $6 \mathrm{~nm}$ nanocrystals. The shift can be accurately determined by deconvolution of the spectra using four Voigt functions corresponding to elastic, $d-d$ loss peaks, and MLCT peaks, as shown in Figure 4b. The total fit is excellent and indistinguishable from the experimental data within the noise level. The charge-transfer peak was found to be at $-7.3 \mathrm{eV}$ for the $9 \mathrm{~nm}$ Co nanocrystals and at $-6.7 \mathrm{eV}$ for $6 \mathrm{~nm}$ and smaller ones. The reference spectra of $\mathrm{CoO}$ and $\mathrm{CoCl}_{2}$ in Figure $4 \mathrm{~b}$ provide a clue as to the cause to the shift. The CT peak in the $9 \mathrm{~nm}$ Co nanocrystals at $-7.3 \mathrm{eV}$ is similar to that of $\mathrm{CoO}$, indicating a strong interaction between Co nanocrystals and the carboxyl group of the oleic acid surfactant. For the smaller Co nanocrystals (3-6 nm), the CT peak is found at $-6.7 \mathrm{eV}$, that is, close to $\mathrm{CoCl}_{2}$, which could be the result of penetration of chlorobenzene molecules through the surfactant shell in the smaller nanocrystals due to less efficient packing. The width of CT peaks in the Co nanocrystals is also narrower than that of the $\mathrm{CoO}$ and close to that of $\mathrm{CoCl}_{2}$, supporting again that the Co nanocrystals interact with the surrounding $\mathrm{C}_{6} \mathrm{H}_{4} \mathrm{Cl}_{2}$ solvent molecules.

In conclusion, we have performed XAS, XES, and RIXS studies of Co nanocrystals. The experimental and theoretical spectra show that the interaction between Co nanocrystals and surfactant and solvent molecules can be measured by in situ techniques, opening the way for in situ studies of growth and reactivity. Our results suggests that the nanocrystals interact more strongly with solvent molecules in the initial stages of growth, while at a later stage, the interaction is dominated by the oleic acid surfactant.

Acknowledgment. Work was supported by the Office of Basic Energy Science, Chemical Sciences Division of the U.S. Department of Energy under contract no. DE-AC02$05 \mathrm{CH} 11231$.

\section{References}

(1) Somorjai, G. A.; Borodko, Y. G. Catal. Lett. 2001, 76, 1-5

(2) Konya, Z.; Puntes, V. F.; Kiricsi, I.; Zhu, J.; Alivisatos, A. P.; Somorjai, G. A. Catal. Lett. 2002, 81, 137-140.

(3) Puntes, V. F.; Krishnan, K. M.; Alivisatos, A. P. Science 2001, 291, 2115-2117.

(4) Puntes, V. F.; Gorostiza, P.; Aruguete, D. M.; Bastus, N. G.; Alivisatos, A. P. Nat. Mater. 2004, 3, 263-268.

(5) Puntes, V. F.; Krishnan, K. M.; Alivisatos, P. Appl. Phys. Lett. 2001, 78, 2187-2189.

(6) Dinega, D. P.; Bawendi, M. G. Angew. Chem., Int. Ed. 1999, 38, $1788-1791$.

(7) de Groot, F. M. F. Chem. Rev. 2001, 101, 1779-1808.

(8) Butorin, S. M.; Guo, J.-H.; Wassdahl, N.; Nordgren, J. E. J. Electron. Spectrosc. Relat. Phenom. 2000, 110-111, 235-273.

(9) Shin, S.; Kotani, A. Rev. Mod. Phys. 2001, 73, 203-246.

(10) Guo, J.-H.; Luo, Y.; Augustsson, A.; Rubensson, J.-E.; Sathe, C.; Agren, H.; Siegbahn, H.; Nordgren, J. Phys. Rev. Lett. 2002, 89, 137402-1-137402-4.

(11) Guo, J.-H.; Luo, Y.; Augustsson, A.; Kashtanov, S.; Rubensson, J.E.; Shuh, D. K.; Agren, H.; Nordgren, J. Phys. Rev. Lett. 2003, 91 , 157401-1-157401-4

(12) Duda, L.-C.; Schmitt, T.; Augustsson, A.; Nordgren, J. J. Alloys Compd. 2004, 362, 116-123.

(13) Nordgren, J.; Guo, J.-H. J. Electron. Spectrosc. Relat. Phenom. 2000 , $110-111,1-13$.

(14) Guo, J.-H.; Augustsson, A.; Englund, C.-J.; Nordgren, J. AIP Conf. Proc. 2004, 705, 1066-1069.

(15) Arrio, M.-A.; Sainctavit, Ph.; dit Moulin, Ch. C.; Mallah, T.; Verdaguer, M.; Pellegrin, E.; Chen, C. T. J. Am. Chem. Soc. 1996, $118,6422-6427$

(16) Hatsui, T.; Takata, Y.; Kosugi, N. Chem. Phys. Lett. 1998, 284, 320324.

(17) Takata, Y.; Hatsui, T.; Kosugi, N. J. Electron. Spectrosc. Relat. Phenom. 1999, 101-103, 443-447.

(18) dit Moulin, C. C.; Villain, F.; Bleuzen, A.; Arrio, M.-A; Sainctavit, P.; Lomenech, C.; Escax, V.; Baudelet, F.; Dartyge, E.; Gallet, J. J.; Verdaguer, M. J. Am. Chem. Soc. 2000, 122, 6653-6658.

(19) Ruehl, E.; Hitchcock, A. P. J. Am. Chem. Soc. 1989, 111, 2614.

(20) Hocking, R. K.; Wasinger, E. C.; Yan, Y.-L.; deGroot, F. M. F.; Walker, F. A.; Hodgson, K. O.; Hedman, B.; Solomon, E. I. J. Am. Chem. Soc. 2007, 129, 113.

(21) Butorin, S. M.; Guo, J.-H.; Magnuson, M.; Kuiper, P.; Nordgren, J. Phys. Rev. B 1996, 54, 4405-4408.

(22) Magnuson, M.; Butorin, S. M.; Guo, J.-H.; Nordgren, J. Phys. Rev. B 2002, 65, 205106-1-205106-5.

(23) Cowan, R. D. The Theory of Atomic Structure and Spectra; University of California Press: Berkeley, 1981; Cowan, R. D. J. Opt. Soc. Am. 1968, 58, 808.

(24) Butler, P. H. Point Group Symmetry, Applications, Methods and Tables; Plenum: New York, 1991.

(25) de Groot, F. M. F. Coord. Chem. Rev. 2005, 249, 31

(26) The main ionic component of the ground state of Co metal is $4 \mathrm{~s}^{2}$ $3 d^{7}$. Within the charge transfer model one would need to add $3 d^{7} 4-$ $(\mathrm{sp})^{2}+3 \mathrm{~d}^{8} 4(\mathrm{sp})^{1}+3 \mathrm{~d}^{9} 4(\mathrm{sp})^{0}\left(+\right.$ possibly $\left.3 \mathrm{~d}^{6} 4(\mathrm{sp})^{3}\right)$.

NL0705860 